\title{
A comparison of skeletal maturity assessed by radiological and ultrasonic methods
}

\begin{tabular}{|r|l|}
\hline Journal: & American Journal of Human Biology \\
\hline Manuscript ID & AJHB-16-0172.R1 \\
\hline Wiley - Manuscript type: & Original Research Article \\
\hline Date Submitted by the Author: & n/a \\
\hline Complete List of Authors: & $\begin{array}{l}\text { Utczas, Katinka; Eotvos Lorand University, Department of Biological } \\
\text { Anthropology } \\
\text { Muzsnai, Agota; Saint Janos Hospital and Unified Hospitals of North Buda, } \\
\text { Pediatric Endocrinology } \\
\text { Cameron, Noel; Loughborough University, Human Sciences } \\
\text { Zsakai, Annamaria; Eotvos Lorand University, Department of Biological } \\
\text { Anthropology } \\
\text { Bodzsar, Eva; Eotvos Lorand University, Department of Biological } \\
\text { Anthropology }\end{array}$ \\
\hline Keywords: & $\begin{array}{l}\text { Skeletal maturity assessment, Greulich-Pyle method, Tanner-Whitehouse } \\
\text { methods, Ultrasonic bone age estimation }\end{array}$ \\
\hline &
\end{tabular}

SCHOLARONE ${ }^{m}$

Manuscripts 


\title{
A comparison of skeletal maturity assessed by radiological and ultrasonic methods
}

\author{
Abbreviated title: Radiological and ultrasonic bone age estimation \\ Katinka Utczas ${ }^{1}$, Agota Muzsnai ${ }^{2}$, Noel Cameron $^{3}$, Annamaria Zsakai $^{1}$, Eva B Bodzsar ${ }^{1}$ \\ ${ }^{1}$ Department of Biological Anthropology, Eotvos Lorand University, 1117 Budapest, Hungary \\ ${ }^{2}$ Department of Paediatric Endocrinology, Saint Janos Hospital and Unified Hospitals of \\ North Buda, Budapest, 1125 Hungary \\ ${ }^{3}$ School of Sport, Exercise and Health Sciences, Loughborough University, LE11 3TU \\ Leicestershire, Great Britain
}

\section{Corresponding author:}

Annamaria Zsakai

Department of Biological Anthropology,

Eotvos Lorand University,

Pazmany P. s. $1 / \mathrm{c}$,

1117 Budapest, Hungary

Tel.: +36-1-381-2161, Fax: +36-1-381-2162

E-mail: zsakaia@elte.hu

Number of text pages: 7 pages +2 pages bibliography

Number of tables and figures: 3 figures and 3 tables

Acknowledgement: The work of Annamaria Zsakai was supported by the Janos Bolyai Research Scholarship (2014-2017) of the Hungarian Academy of Sciences. The authors report no conflicts of interest. 


\begin{abstract}
Objectives: The estimation of skeletal maturity is a useful tool in paediatric practice to determine the degree of delay or advancement in growth disorders and the effectiveness of treatment in conditions that influence linear growth. Skeletal maturity of children is commonly assessed by using either Greulich-Pyle (GP) or Tanner-Whitehouse methods (TW2 and TW3). However, a less invasive ultrasonic method, that does not use ionizing radiation, has been suggested for use in epidemiological studies of skeletal maturity. The main purpose of the present study was to determine the accuracy of an ultrasonic method based on the GP maturity indicators compared to the standard GP radiographic method.

Methods: Skeletal maturity of 1502 healthy children, aged from 6 to 18 yrs, was estimated by quantitative ultrasound and compared to GP bone ages estimated from left hand and wrist radiographs of a subsample of 47 randomly selected participants.

Results: The ultrasonic bone age estimation demonstrated very strong correlations with all the radiological age estimations. The correlation coefficients ranged between 0.895 and 0.958 , the strongest correlation of ultrasonic skeletal maturity estimation was found with the TannerWhitehouse RUS method. The ultrasonic bone age estimation is suggested to use between the chronological ages 8.5-16.0 years in the boys and 7.5-15.0 years in the girls.

Conclusions: The ultrasonic bone age estimation is suggested to be used in epidemiological surveys since the sensitivity for screening not normal bone development is appropriate at least within the $8-15$ years age interval.
\end{abstract}

Keywords: Skeletal maturity assessment, Greulich-Pyle method, Tanner-Whitehouse methods, Ultrasonic bone age estimation 


\section{Introduction}

Biological ages are usually estimated from the developmental and maturity status and/or physiological functioning of specific organ systems (e.g. skeletal, reproductive, endocrine, neurological, etc.). The relationship between biological and chronological age provides information on the rate of maturation or tempo of growth (Cole et al., 1988; Vallej-Bolanos et al., 1999). Skeletal, dental and morphological ages are the most commonly used biological ages in growth studies (Bodzsar and Susanne, 2000; Gasser et al., 2013).

Skeletal maturity of children within the context of suspected growth disorders is usually determined in clinical practice using either the Tanner-Whitehouse "bone-specific scoring" method (TW) or the Greulich-Pyle "atlas system" (GP; Martin et al., 2011). Both methods (1) are based on the radiographs of the wrist and hand region of the skeleton, (2) estimate the bone age of children on the basis of the maturity indicators of selected bones from this region, (3) are performed by using minimal radiation exposure on multiple ossification centres (Bull et al., 1999; Ahmed and Warner, 2007; Martin et al., 2011).

The advantage of the TW method (Tanner et al., 1983, 2001) is that the it gives a possibility to consider only the round bones (carpus; CA) or only the radius, ulna and "short" bones (radius, ulna, metacarpals and phalanges; RUS) bones, or both types of bones together in the bone age estimation (TW20, but only in TW2 method). While Greulich and Pyle (1971) recommended that each bone in the hand and wrist be compared to a series of maturity indicators this procedure is rarely used. Instead the vast majority of practitioners simply compare the radiograph of interest with the atlas standards presented by gender and chronological age.

However, the minimal level of radiation currently required for hand-wrist radiographs is $0.003 \mathrm{mSv}$ compared to a general annual background radiation of $2.7 \mathrm{mSv}$ in the UK, i.e. $1 / 900^{\text {th }}$ of the normal yearly exposure (Public Health England, 2011). This exposure level is not usually permitted in epidemiological surveys. In such surveys skeletal maturity is usually determined only by considering some anthropometric dimensions e.g. humeral and femoral epicondylar breadths.

Ultrasound is an alternative technique to estimate parameters of skeletal quality by measuring bone structural variables as speed of ultrasound and broadband ultrasonic attenuation through the region of interest. The parameters of bone quality in relation to age-related reference values can provide estimates of skeletal maturity as well. Usually peripheral skeletal sites, such as the calcaneus, phalanges of the hand, ulna, radius and tibia are scanned by ultrasound. 
One of the ultrasonic devices developed for skeletal age evaluation is the BonAge device (Sunlight Medical Ltd., Israel). This device scans the ossifying cartilage structures of the wrist region (ulna and radius), and estimates the bone age of children between the age of 5 and 18 years. The bone age estimation algorithms were constructed by considering the relationship between the ultrasonic parameters and the radiographic GP bone age estimates (Mentzel et al., 2005).

The basic aims of the present analysis were (1) to estimate the accuracy of ultrasonic skeletal maturity estimation made by the BonAge device in a sample of healthy children by comparing US, TW and GP estimates of skeletal maturity from 5-18 years of age (i.e. differences and correlations), and (2) to determine over what age range US can be used as an acceptable estimate of skeletal maturity.

To attain this main aim

- the estimates of Tanner-Whitehouse 2 and 3 methods were compared and by considering the results of the comparison and the international recommendations (Ahmed and Warner 2007) the most recent TW3 method was used in the further analyses.

- The relationship between the bone ages estimated radiographic (TW3 and GP) and ultrasonic methods and chronological age were analysed to describe the accuracy of the ultrasonic method.

- The relationship between the Tanner-Whitehouse and Greulich-Pyle radiographic estimations, were analysed.

- The relationship among the bone age estimation methods made by using the Greulich-Pyle radiologic and the ultrasonic methods was used to specify the chronological age interval of applicability for the studied ultrasonic estimation.

\section{Subjects and methods}

The skeletal maturity of healthy children aged 6-18 years (760 boys and 742 girls) was estimated by a quantitative ultrasound based device, a Sunlight Bone age device. The BonAge quantitative ultrasonic system (Sunlight Medical Ltd.; BA) measures bone acoustic parameters at the wrist and provides an accurate estimation of bone maturity that correlates with Greulich-Pyle readings. The absolute average difference between BA and GP estimates were: 0.90 years in boys and 0.86 in girls, and correlations between BA and GP were: 0.95 in boys, 0.94 in girls (Zadik et al., 2003; Mentzel et al., 2005). Each child underwent 3 repeated 
readings of ultrasonic bone age assessment and the mean of the 3 measurements was used in the analyses (GPB).

In a randomly selected subsample (n: 47, the financial capabilities of the research and the methodological difficulties limited the number of subjects in this subsample) the bone age of children (aged between 7.0 and $17.5 \mathrm{yrs}$ ) was also determined from standard radiographs of the left hand and wrist region by the GP and TW3 (using TW3 RUS and CARP in each case). Hand X-ray scans were performed in the Saint Janos Hospital and Unified Hospitals of North Buda, Budapest, Hungary. All scans were assessed separately and individually, by one paediatric endocrinologist (AM) and by two trained human biologists (NC and KU) without their knowledge of the bone age assessment by the other investigators. Disagreements were solved by discussion. Bone age assessments were blindly assessed according to chronological age. The Cohen's Kappa values of inter-assessor agreements ranged between 0.886 and 1.000 in the Tanner-Whitehouse method, while between 0.943 and 1.000 in the Greulich-Pyle method (this means in general that less than 5 children's rating in TW3 method and less than 3 children's rating in GP method differed between two assessors). Estimated stages of each bone were used in the inter-observer accordance tests and not the total maturity scores of individuals to avoid the bias arising from summing up the maturity scores of individual bones. This indicated a very good inter-assessor agreement, which suggests and confirms a very good applicability the studied two radiologic methods to evaluate skeletal development.

The one sample t test was used to estimate the significance of the difference between bone age estimates. Due to the not normal distribution of the studied age variables in the gender subgroups (Shapiro-Wilk test, $\mathrm{p}<0.05$ ) the Spearman's rho coefficient of rank correlation was estimated to describe the relationship between chronological age and bone age estimations in the studied sample. Linear regression analysis (extended with curvilinear regression analysis which analysis confirmed the linear relation between the studied age variables) was used to study the relationship between radiographic Greulich-Pyle and ultrasonic bone age estimates. Hypotheses were tested at the 5\% level of significance.

Written informed consent was obtained from the legal guardians of children, and assent was obtained from the children as well. The research protocol and procedures employed were reviewed and approved by Research Ethics Committee of Saint Janos Hospital and Unified Hospitals of North Buda. Children with bone diseases or taking any medication which might change bone metabolism or mineralization were excluded from the analysis. 


\section{Results}

The relationships among the chronological age and the bone age estimates

Although it is known that the studied radiologic estimates of skeletal maturity using almost the same maturity indicators, i.e. they ought to show very high correlations, the first step in the analysis was to evaluate this relationship among the bone age estimates - both radiologic and ultrasonic methods - and chronological age in the studied sample to scale the correlations among them. The correlation coefficients between the estimated biological ages and chronological age were very high (ranged between 0.862 and 0.982 ), the strongest relations were found between TW3 RUS, CARP and GP estimates in both genders (Table 1). The correlations between the chronological age and bone ages were weaker in the girls. The ultrasonic bone age estimation (BA) showed very strong correlations with the radiological age estimations in both genders $(r>0.890)$.

[Table 1 here]

The comparison of the Tanner-Whitehouse (TW3) and Greulich-Pyle method estimates

By considering the distribution of differences between individual skeletal age estimations (TW3 RUS, CARP and GP) the following can be stated: (1) the differences from the GP bone age estimates showed a declining tendency with increasing chronological age in the case of CARP age; and (2) the range of the differences between the RUS and GP estimates was stable in both genders, but was weaker in the girls (Fig. 1, Table 2).

[Figure 1 and Table 2 here]

The comparison of bone age estimates by the Greulich-Pyle and the ultrasonic methods

In general the bone age and chronological age do not differ significantly in large samples of healthy children according to the results of auxological studies, e.g. the mean bone age of children aged 10 years is usually around 10 years as well. Therefore the study of BA bone age estimates was started by the visual analysis of BA age - chronological age graphs. The relation of ultrasonic bone age estimates with chronological age revealed that this method can be used for skeletal maturity estimation until the chronological age of 16 years in the boys and until the age of 15 years in the girls, since the pattern of individual BA estimates deviated from the \pm 2 year-wide zone of difference between the BA and chronological age (Fig. 2, 
1

2

3

4

5

6

7

8

9

10

11

12

13

14

15

16

17

18

19

20

21

22

23

24

25

26

27

28

29

30

31

32

33

34

35

36

37

38

39

40

41

42

43

44

45

46

47

48

49

50

51

52

53

54

55

56

57

58

59

60

Table 3). This difference between any biological age and chronological age is considered to be normal in healthy children in auxological studies (Vallejo-Bolanos et al. 1999, Tanner et al. 2001; in forensic studies bigger population specific differences have already been demonstrated between mean chronological and bone ages, but usually through the whole subadult phase of human life cycle and not only in small age intervals of infant or juvenile phases, e.g. Schmeling et al. 2000, Buken at al. 2007).

By comparing the BA estimates to the GP estimates (Fig. 3) it could be stated that the ultrasonic bone age estimation (BA) gave a very good estimation of GP bone ages when the age interval was narrowed to 7.5 years in the girls and 8.5 years in the boys (the ultrasonic method estimated the skeletal maturity with very small error, the $\beta_{1}$ parameter of the linear regression between GP and BA approached the 1.000 value and the $\beta_{2}$ parameters the 0.000 expected value of total equality, Fig. 3).

[Figures 2-3 and Table 3 here] 


\section{Discussion}

Estimates of skeletal maturity are generally performed only in the clinical practice (Mahaney, 1991; Aicardi et al. 2000). Since the conventional methods of skeletal maturity estimations are based on X-ray scans and due to the ionizing radiation these methods cannot be used in epidemiological screening surveys. The possible alternative method to the radiographic method could be the ultrasonography-based method, however the ultrasound techniques in skeletal maturity estimation has not been widely used either in the epidemiological or in the clinical practice. The main purpose of this study was to examine the applicability the ultrasonic Sunlight BonAge device for skeletal maturity determination in healthy children.

The ultrasonic Sunlight BonAge bone age estimates showed very strong correlations with the radiographic estimates. This observation verified the suitability of ultrasonic Sunlight BonAge device in skeletal maturity estimation. Although the device was introduced more than 10 years ago, this is the first systematic comparison of bone age estimates based on x-ray scans and the Sunlight BonAge estimates.

Although the inventors (Mentzel et al., 2005) introduced the novel device for skeletal age assessment between 6 and 17 years of age in both genders, on the basis of the present analysis the chronological age interval between 8.5-16 years in the boys and 7.5-15 years in the girls is recommended for skeletal maturity estimation by the ultrasonic Sunlight BonAge device.

\section{Conclusions}

By considering the analysis of radiologic and ultrasonic bone age estimations in the studied sample of Hungarian children aged between 6-18 years it can be concluded that:

- the accuracy of the studied ultrasonic method showed strong correlations with all the standard radiographic methods.

- The GP method showed very strong correlation with both the TW3 RUS and TW3 CARP bone age estimations. However, the analysis of the individual differences between the GP and TW3 estimates revealed that the differences between the two radiological age estimations were stable between RUS and GP, while CA had a significant linear (decreasing) tendency with GP by chronological age in both genders. This decreasing tendency of CA bone age with GP estimates from the age of 12 years can be explained by 
the different pattern of carpal maturation, i.e. the earlier cessation of development of carpal bones (ossa brevia, short bones).

- By considering the comparison of ultrasonic and radiologic bone age estimates the ultrasonic bone age estimation (with the Sunlight BonAge device) is suggested to use between the chronological ages 8.5-16 years in the boys and 7.5-15 years in the girls.

In conclusion, the presented results confirmed that the Greulich-Pyle radiological method of bone age estimation can be used instead of the more accurate, but more time-consuming Tanner-Whitehouse method in the clinical practice, since the bone age estimates showed very strong correlations between these bone age estimates. However, TW bone age estimations, its both types: the carpal and the RUS estimations are suggested to use when the accuracy of the skeletal maturity assessment takes a key role in the diagnosis of growth disorders, or the RUS estimates are suggested to use from the age interval as the carpal bones reach their final development. The very strong correlation of radiological and ultrasonic bone age estimates gives us the possibility (1) to use the ultrasonic bone age estimation in epidemiological surveys to assess skeletal maturity in children without the use of ionizing radiation, and (2) to introduce new equations for skeletal maturity development based only on anthropometric measurements that equations will be constructed in our former analysis of ultrasonic bone age assessments and body development in the studied sample.

Acknowledgement: The work of Annamaria Zsakai was supported by the Janos Bolyai Research Scholarship (2014-2017) of the Hungarian Academy of Sciences.

Author contributions: KU, AM, AZs and EBB contributed in the fieldworks, x-ray scans were assessed by KU, AM and NC, while all authors contributed in the statistical analysis and in the data interpretation of the presented study.

\section{Literature cited}

Ahmed ML, Warner JT. 2007. TW2 and TW3 bone ages: time to change? Arch Dis Child 92(4):371-372. 
Aicardi G, Vignolo M, Milani S, Naselli A, Magliano P, Garzia P. 2000. Assessment of skeletal maturity of the hand-wrist and knee: A comparison among methods. Am J Hum Biol 12(5):610-615.

Bodzsar EB, Susanne C (Eds). 2000. Puberty: Variability of Changes and Complexity of Factors. Budapest: Eotvos University Press, p 211.

Buken B, Şafak AA, Yazici B, Buken E, Mayda AS. 2007. Is the assessment of bone age by the Greulich-Pyle method reliable at forensic age estimation for Turkish children? Forensic Sci Int 173(2):146-153.

Bull RK, Edwards PD, Kemp PM, Fry S, Hughes IA. 1999. Bone age assessment: a large scale comparison of the Greulich and Pyle, and Tanner and Whitehouse (TW2) methods. Arch Dis Child 81(2):172-173.

Cole AJ, Webb LL, Cole TJ. 1988. Bone age estimation: a comparison of methods. Brit J Radiol 61(728):683-686.

Gasser T, Molinari L, Largo R. 2013. A comparison of pubertal maturity and growth. Ann Hum Biol 40(4):341-347.

Greulich WW, Pyle SI, Waterhouse AM. 1971. A radiographic standard of reference for the growing hand and wrist. Chicago: Case Western Reserve University.

Mahaney MC. 1991. Differentially delayed development in the hand-wrist skeletons of children with cystic fibrosis: Round versus tubular bones. Am J Hum Biol 3(1):17-24.

Martin DD, Wit JM, Hochberg ZE, Savendahl L, Van Rijn RR, Fricke O, Cameron N, Caliebe J, Hertel T, Kiepe D, Albertsson-Wikland K, Thodberg HH, Binder G, Ranke MB. 2011. The use of bone age in clinical practice - part 1. Horm Res Paediatr 76(1):1-9.

Mentzel HJ, Vilser C, Eulenstein M, Schwartz T, Vogt S, Böttcher J, Yaniv I, Tsore L, Kauf E, Kaiser WA. 2005. Assessment of skeletal age at the wrist in children with a new ultrasound device. Pediatr Radiol 35(4):429-433.

Public Health England. 2011. Ionising radiation: dose comparisons. Published 18 March 2011 https://www.gov.uk/government/publications/ionising-radiation-dosecomparisons/ionising-radiation-dose-comparisons (accessed 01/10/15)

Schmeling A, Reisinger W, Loreck D, Vendura K, Markus W, Geserick G. 2000. Effects of ethnicity on skeletal maturation: consequences for forensic age estimations. International J Legal Med 113(5):253-258.

Tanner JM, Healy M, Goldstein H, Cameron N. 2001. Assessment of skeletal maturity and prediction of adult height (TW3 Method), 3rd edn. London: WB Saunders, Harcourt Publishers Ltd 2001. 
Tanner JM, Whitehouse RH, Cameron N, Marshall WA, Healy MUR, Goldstein H. 1983. Assessment of skeletal maturity and prediction of adult height (TW2 Method). 2nd edn. London: Academic Press.

Vallejo-Bolanos E, Espana-Lopez AJ, Munoz-Hoyos A, Fernandez-Garcia JM. 1999. The relationship between bone age, chronological age and dental age in children with isolated growth hormone deficiency. Int J Paediatr Dent 9(3):201-206.

Zadik Z, Bistrizer T, Tsoref L, Schwartz T, Yaniv I. 2003. A Novel Method for Assessing Bone Age Using Ultrasound. Europediatrics, Prague. Programma and Abstracts. 
Table 1. Spearman's rho coefficients of rank correlations ( $p<0.001$ for every correlation) between the estimated bone ages (CA: chronological age, RUS and CARP: TW3 bone ages, GP: Greulich-Pyle bone age, BA: bone age estimated by the ultrasound device)

\begin{tabular}{|c|c|c|c|c|c|c|}
\hline & \multicolumn{5}{|c|}{ Boys } \\
\hline & & $\mathrm{CA}$ & RUS & CARP & GP & BA \\
\hline \multirow{5}{*}{$\stackrel{n}{\Xi}$} & $\mathrm{CA}$ & & 0.958 & 0.944 & 0.957 & 0.921 \\
\hline & RUS & 0.862 & & 0.941 & 0.964 & 0.891 \\
\hline & CARP & 0.896 & 0.972 & & 0.982 & 0.936 \\
\hline & GP & 0.864 & 0.981 & 0.975 & & 0.944 \\
\hline & BA & 0.859 & 0.931 & 0.925 & 0.893 & \\
\hline
\end{tabular}


2

3

4

5

6

7

8

9

10

11

12

13

14

15

16

17

18

19

20

21

22

23

24

25

26

27

28

29

30

31

32

33

34

35

36

37

38

39

40

41

42

43

44

45

46

47

48

49

50

51

52

53

54

55

56

57

58

59

60

Table 2. The statistical parameters of differences between individual skeletal age estimations (Tanner-Whitehouse estimates: TW3 RUS and CARP; Greulich-Pyle estimates: GP; CI: 95\% confidence interval; \%: Percentages of paired measurements showing a tendency to provide concordant results, i.e. the ratio of individuals with the absolute difference not higher than $1.0)$

\begin{tabular}{lcccc}
\hline & \multicolumn{2}{c}{ RUS-GP } & \multicolumn{2}{c}{ CA-GP } \\
& Boys & Girls & Boys & Girls \\
\hline Mean & 0.11 & 0.02 & -0.16 & -0.66 \\
SD & 1.09 & 0.60 & 0.99 & 1.58 \\
SE & 0.21 & 0.14 & 0.19 & 0.36 \\
CI & $-0.31-0.54$ & $-0.27-0.31$ & $-0.54-0.23$ & $-1.40--0.01$ \\
$\%$ & 64.29 & 89.47 & 67.86 & 52.63 \\
\hline
\end{tabular}


Table 3. The statistical parameters of differences between individual skeletal age estimations (GP: Greulich-Pyle radiographic estimates, BA: Greulich-Pyle ultrasonographic estimates; *: estimates for the narrowed chronological age interval: 7.5-15 years in the girls, 8.5-16 years in the boys; CI: 95\% confidence interval; \%: Percentages of paired measurements showing a tendency to provide concordant results, i.e. the ratio of individuals with the absolute difference not higher than 1.0)

\begin{tabular}{lcccc}
\hline & \multicolumn{2}{c}{ BA-GP } & \multicolumn{2}{c}{ BA-GP* } \\
& Boys & Girls & Boys & Girls \\
\hline Mean & -0.16 & -0.16 & -0.12 & -0.15 \\
SD & 1.34 & 1.34 & 1.35 & 1.09 \\
SE & 0.31 & 0.31 & 0.29 & 0.29 \\
CI & $-0.72-0.44$ & $-0.72-0.44$ & $-0.63-0.60$ & $-0.78-0.47$ \\
$\%$ & 57.14 & 57.14 & 52.38 & 64.29 \\
\hline
\end{tabular}


Fig. 1. Differences between individual skeletal age estimations (Tanner-Whitehouse estimates: TW3 RUS and CARP; Greulich-Pyle estimates: GP; n: Boys, ○: Girls)

Fig. 2. Individual ultrasonic bone age estimates (GPB) in children aged between 6 and 18 years and in a narrowed age interval (between 8.5-16.0 years in the boys and between 7.515.0 years in the girls) - the dashed lines represent the chronological age \pm 2 years interval (: chronological age - bone age $=0$ )

Fig. 3. GP vs. BA individual skeletal age estimates (A: in the whole subsample, B: in a narrowed sample; $\mathbf{m}$ : Boys, $\bigcirc$ : Girls, - : linear regression between GP and BA in boys, A: $\mathrm{GP}=1.157 \times \mathrm{BA}+1.943, \mathrm{~B}: \mathrm{GP}=1.011 \times \mathrm{BA}+0.029,-$ : linear regression between $\mathrm{GP}$ and $\mathrm{BA}$ in girls, $\mathrm{A}: \mathrm{GP}=1.023 \times \mathrm{BA}-0.204, \mathrm{~B}: \mathrm{GP}=1.081 \times \mathrm{BA}+0.149$ ) 


4
4
5
5
5
5

4
5
5
5
5

4
5
5
5
5

5
5
5
5
5
6

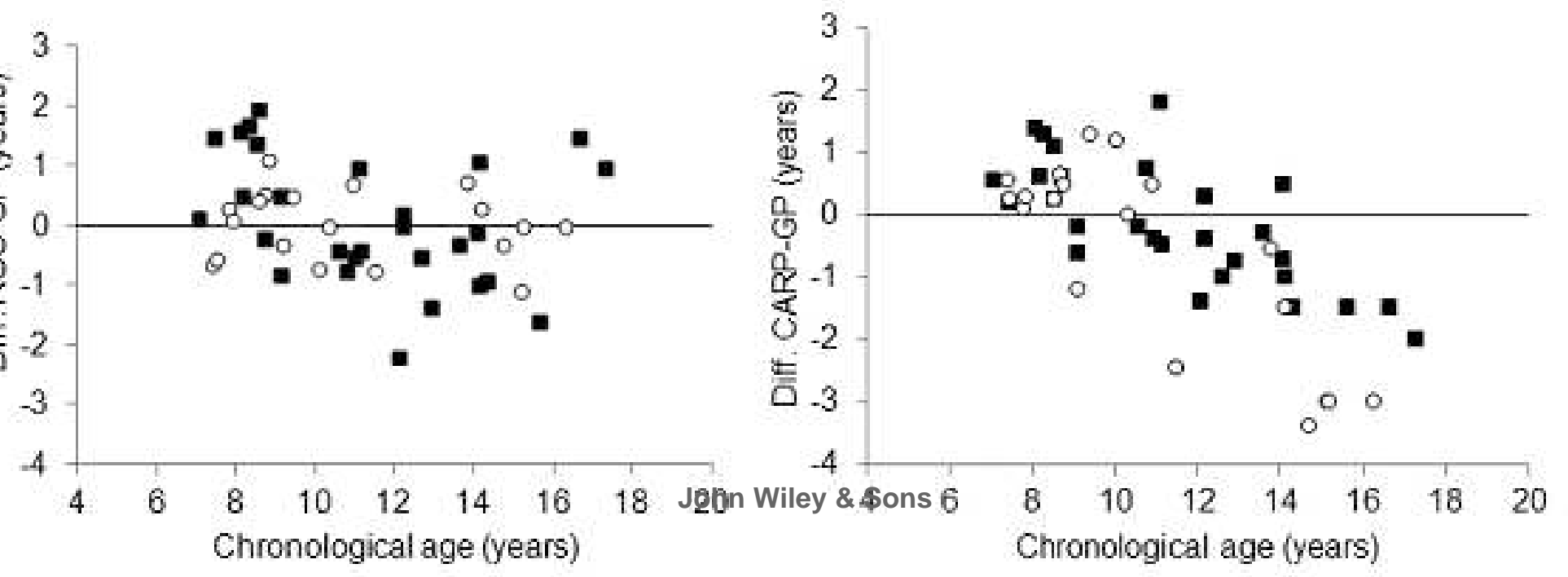




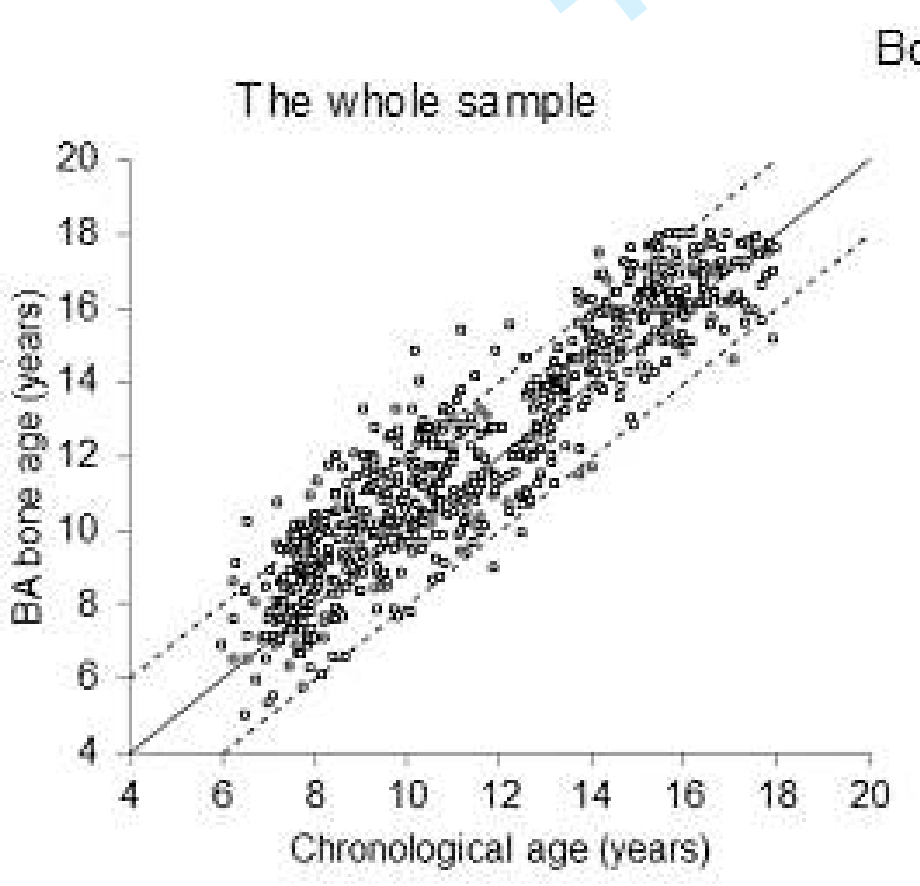

Boys
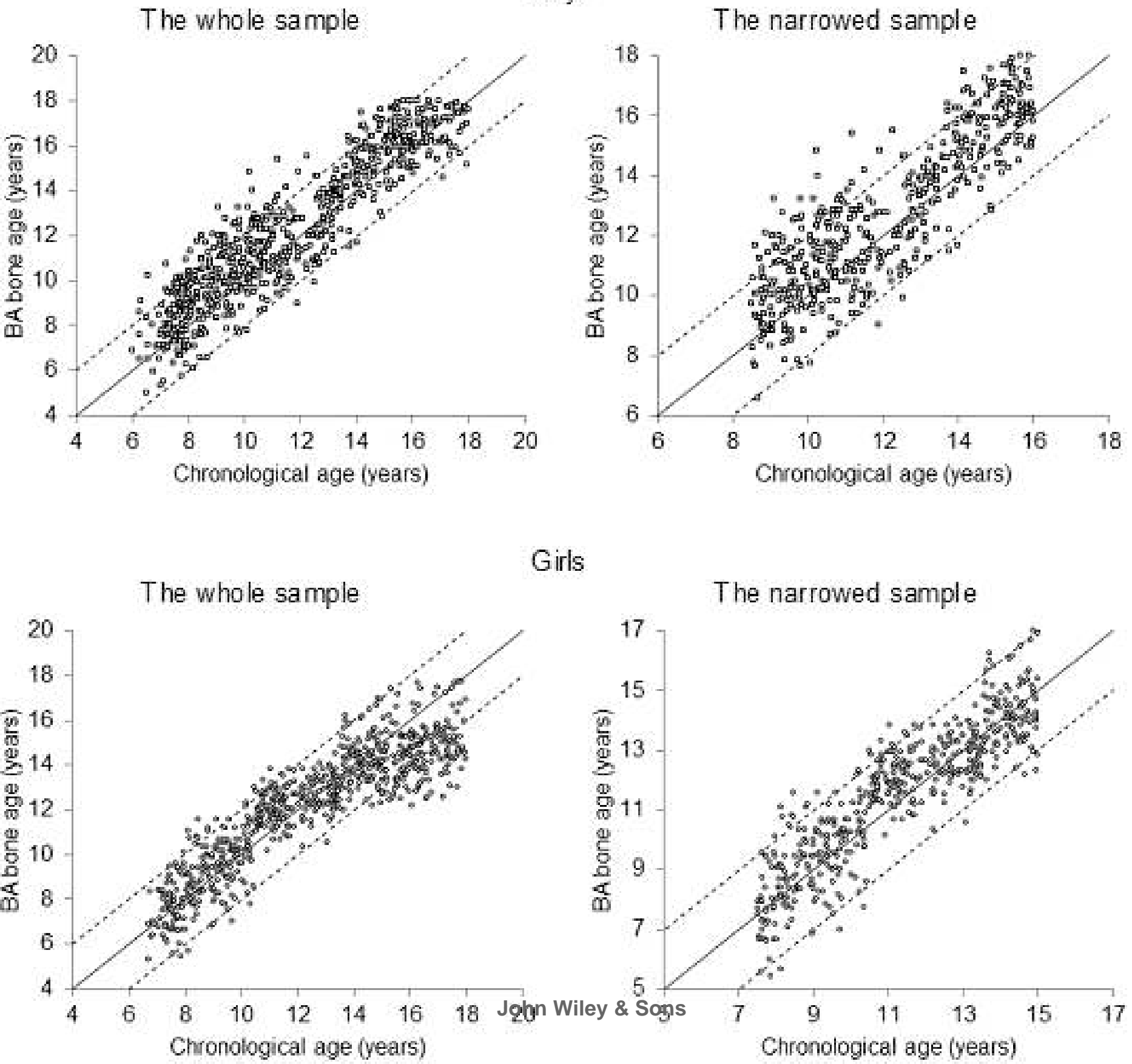

Girls

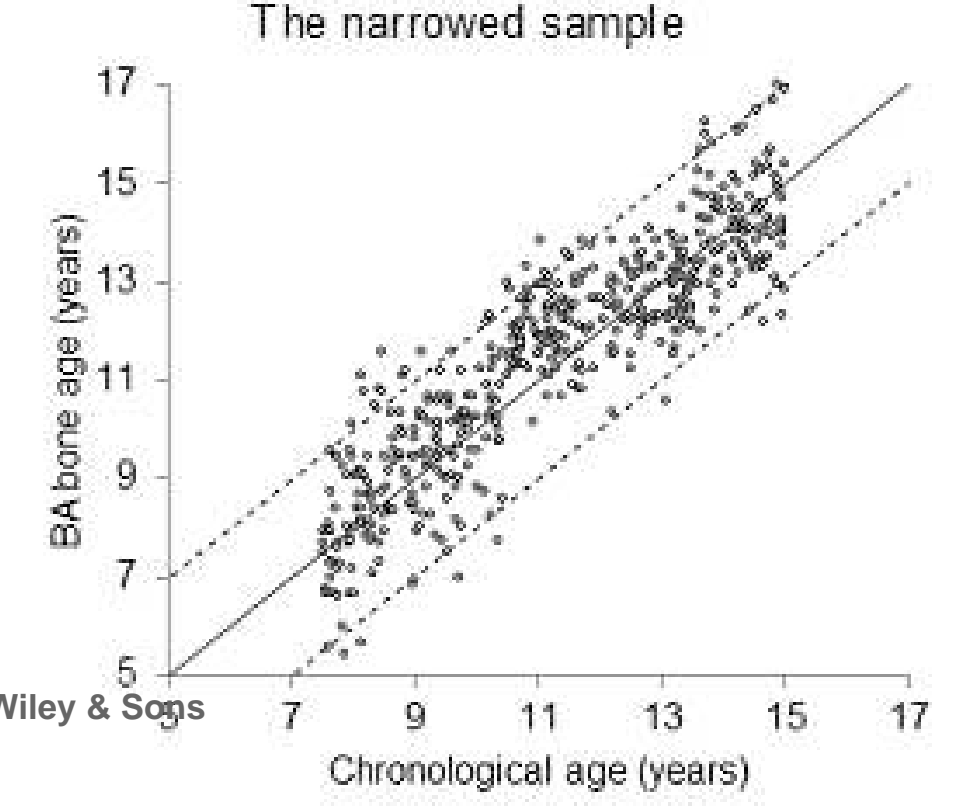


A
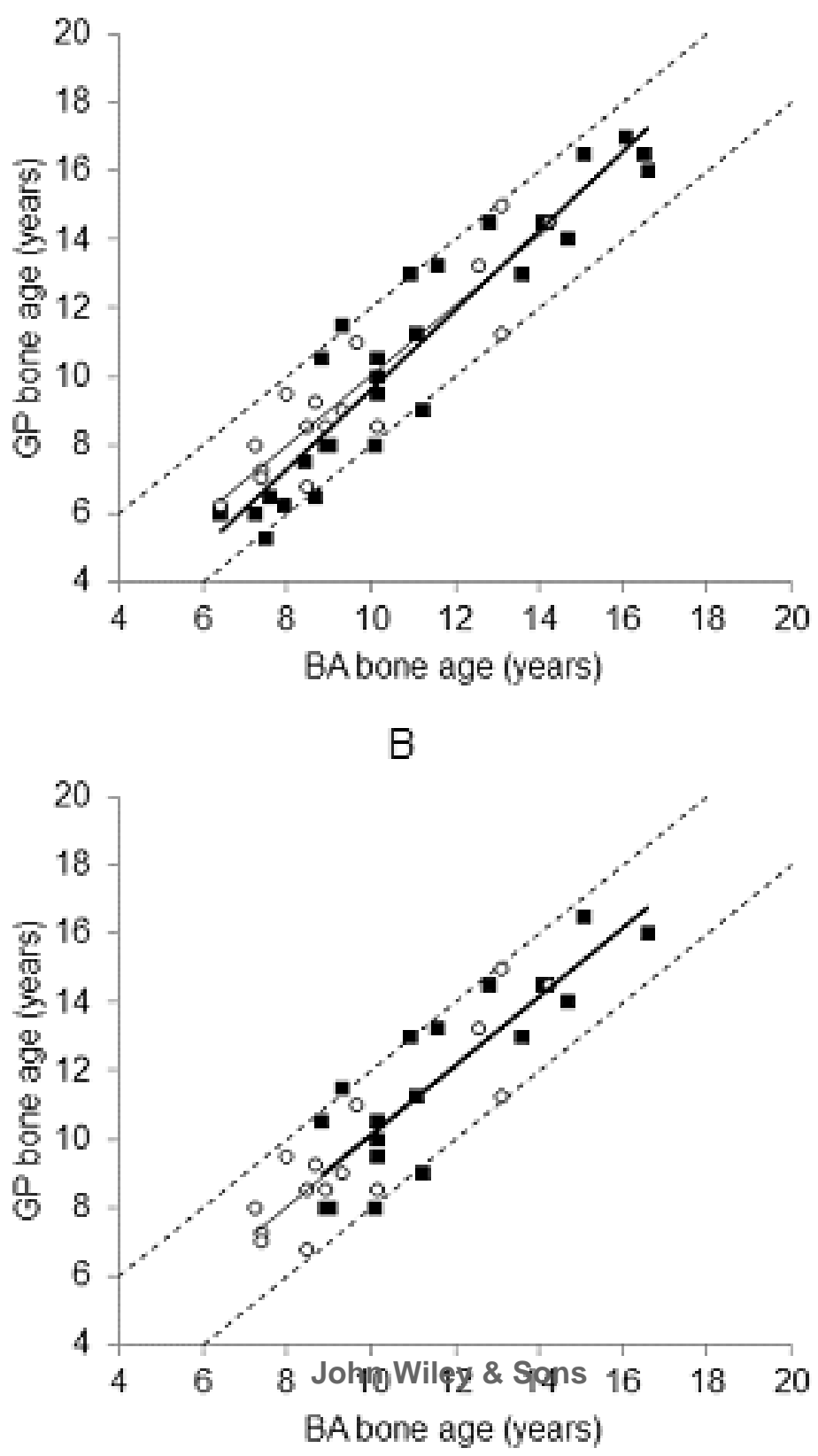